\title{
Composição florística e distribuição de Orchidaceae em uma mata ciliar no Rio Grande do Sul
}

\author{
Floristic composition and distribution of Orchidaceae \\ in a riparian forest in Rio Grande do Sul state
}

Viviane Pagnussat Klein ${ }^{1,5}$, Vivairo Zago ${ }^{2}$, Cristiano Roberto Buzatto ${ }^{3}$ \& Raquel Lüdtke

\begin{abstract}
Resumo
Matas ciliares são ambientes que apresentam condições favoráveis para o estabelecimento de Orchidaceae, no entanto, diferenças no gradiente altitudinal e alterações na conservação destes ambientes podem influenciar as espécies presentes. Objetivou-se verificar a composição florística e distribuição de Orchidaceae ocorrentes na mata ciliar do Arroio Canhada Funda em Pouso Novo, Rio Grande do Sul. Cinco pontos foram marcados ao longo do arroio em diferentes altitudes para a realização das coletas, as quais foram efetuadas utilizando-se o Método do Caminhamento. Com medidas de dissimilaridade e análise de agrupamento verificou-se a similaridade florística entre os pontos, e a relação entre riqueza e altitude foi analisada por correlação linear de Pearson. Foram inventariadas 36 espécies e 23 gêneros, sendo Gomesa (5), Acianthera (4) e Cyclopogon (4) os mais representativos. A maior diversidade foi observada em altitudes inferiores a $300 \mathrm{~m}$ e onde a vegetação estava mais preservada. Acredita-se que a composição e distribuição de Orchidaceae no local foi influenciada principalmente pelo gradiente altitudinal e pelas condições de conservação da mata ciliar.
\end{abstract}

Palavras-chave: floresta ribeirinha, gradiente altitudinal, gradiente de degradação, similaridade florística.

\begin{abstract}
Riparian forests are known to be a favorable environment for the establishment of Orchidaceae, however, difference in altitudinal gradient and changes in environmental conditions affect species diversity. This study aimed to investigate the composition and distribution of Orchidaceae occurrence in a riparian forest of the river Canhada Funda in Pouso Novo, Rio Grande do Sul. Five sampling points were marked along the stream and the species where sampled by the "Caminhamento" method. With measures of dissimilarity and clustering analysis the floristic similarity between the points was verified and the relation between species richness and altitud was verified by Pearson's linear correlation. Thirty six (36) species and 23 genera were inventoried, being Gomesa (5), Acianthera (4) and Cyclopogon (4) the most representative. The greatest diversity of orchids was observed at elevations below $300 \mathrm{~m}$ were vegetation was more preserved. It is believed that the composition and distribution of Orchidaceae in the area was influenced mainly by the altitudinal gradient and the conservation conditions of the riparian forest.

Key words: riparian forest, altitudinal gradient, degradation gradient, floristic similarity.
\end{abstract}

\section{Introdução}

Orchidaceae Juss. é uma das famílias mais representativas e diversificadas de Angiospermas, apresentando cerca de 26.000 espécies e 736 gêneros (Chase et al. 2015). O Brasil detém a terceira maior diversidade de Orchidaceae das Américas, sendo relatados 236 gêneros e aproximadamente 2.548 espécies, das quais 1.636

\footnotetext{
${ }^{1}$ Universidade Federal de Pelotas, Inst. Biologia, Depto. Botânica, Campus Universitário Capão do Leão, C.P. 354, 96010-900, Pelotas, RS, Brasil. vi-klein@) hotmail.com

${ }^{2}$ Emater-RS, R. Coronel Falkemback 181, Bairro Fontes, 99300-000, Soledade, RS, Brasil. vivairo@yahoo.com.br

${ }^{3}$ Universidade de Passo Fundo, Inst. Ciências Biológicas, Prog. Pós-graduação em Ciências Ambientais, Campus I, Bairro São José, BR-285, 99052-900, Passo Fundo, RS, Brasil. crbuzatto@gmail.com

${ }^{4}$ Universidade Federal de Pelotas, Inst. Biologia, Depto. Botânica, Campus Universitário Capão do Leão, 96010-900, Pelotas, RS, Brasil. raquelludtke28@, gmail.com

${ }^{5}$ Autor para correspondência: vi-klein@hotmail.com
} 
são endêmicas (BFG 2015). Fatores ecológicos, condições climáticas e edáficas moldam a estrutura e distribuição da vegetação, influenciando, desta maneira, também a composição e diversidade de espécies de orquídeas (Johansson 1974; Jacquemyn et al. 2005; Ding et al. 2016). No Brasil, em regiões ao sul do paralelo $30^{\circ}$ é observada redução na riqueza de espécies, sendo relatados 90 gêneros e 360 espécies para o Rio Grande do Sul (Waechter 1998; Buzatto et al. 2007; BFG 2015).

Espécies de Orchidaceae são representativas em ambientes conservados que apresentem temperaturas elevadas combinadas com alta umidade atmosférica, sendo comuns em locais próximos a cursos de água (Benzing 1990). Desta forma, as matas ciliares (também conhecidas como florestas ribeirinhas ou matas de galerias) são formações vegetais que circundam córregos, rios, lagos e corpos de água e consistem em um ecossistema peculiar que favorece o desenvolvimento destes táxons (Vogel et al. 2009). No entanto, devido a uma série de fatores como a intensificação agrícola, reflorestamento com espécies exóticas e a pecuária intensiva, observa-se um cenário de fragmentação e destruição destes ambientes, o que coloca em risco toda a biodiversidade associada. Estudos avaliando a diversidade de plantas epífitas em diferentes ambientes com algum grau de perturbação antrópica tem revelado que alterações na vegetação florestal tem afetado negativamente a riqueza e abundância da flora epifítica, sobre tudo, aquelas espécies mais sensíveis as mudanças microclimáticas (Barthlott et al. 2001; Krömer \& Gradstein 2003; Wolf 2005; Hietz et al. 2006; Dettke et al. 2008; Bataghin et al. 2010; Bataghin et al. 2017).

Embora muito se questione sobre a conservação dos ambientes ciliares, estudos que expressam a riqueza e a diversidade de espécies de Orchidaceae associadas a estas matas ainda são escassos. No Rio Grande do Sul, entre os trabalhos realizados nestes ambientes, a maioria se deteve em avaliar a composição das plantas epífitas em geral e não especificamente a flora de Orchidaceae (Freitas \& Jasper 2001; Rogalski \& Zanin 2003; Giongo \& Waechter 2004; Jasper et al. 2005). Sobre Orchidaceae, nos últimos anos um número representativo de levantamentos florísticos (Rocha \& Waechter 2006; Buzatto et al. 2007; Brustulin \& Schmitt 2008; Perleberg 2009; Heberle et al. 2012) e estudos taxonômicos (Rocha \& Waechter 2006; Buzatto et al. 2010a; Buzatto et al. 2010b; Buzatto et al. 2014; Pedron et al. 2014), foram realizados, no entanto, muitas regiões e táxons do estado ainda carecem de estudos.

Tendo em vista que as matas ciliares que acompanham os corpos de água correntes, geralmente apresentam variação no estado de conservação da vegetação associada e na altitude ao longo do percurso dos rios, objetivou-se neste trabalho verificar a composição florística e distribuição das espécies de Orchidaceae ocorrentes na mata ciliar às margens do Arroio Canhada Funda em Pouso Novo, Rio Grande do Sul, Brasil.

\section{Material e Métodos}

Área de estudo

$\mathrm{O}$ estudo foi realizado na mata ciliar às margens do Arroio Canhada Funda, localizado no município de Pouso Novo, Rio Grande do Sul (RS) (Fig.1). O município faz parte da região fisiográfica Encosta Inferior do Nordeste, inserido no Domínio Fitogeográfico Floresta Atlântica (IBGE 2011). O Arroio Canhada Funda faz parte da Bacia Hidrográfica Taquari-Anta, localizado na Linha Medorema, possui aproximadamente $6 \mathrm{~km}$ de extensão e largura média de 3,5 m. A nascente está localizada em um ponto com altitude de $574 \mathrm{~m}$ acima do nível do mar e a deságua ocorre no Rio Fão, onde a altitude é de $112 \mathrm{~m}$, sendo observado um gradiente altitudinal, com mais de $400 \mathrm{~m}$ de desnível entre o primeiro e último ponto de coleta. A mata ciliar é formada por vegetação característica da Floresta Estacional Decidual e apresenta variações no seu estado de conservação ao longo do percurso do arroio, sendo também observado um gradiente de degradação (Cordeiro \& Hasenack 2009).

\section{Amostragem}

Para o levantamento das espécies de Orchidaceae presentes na área de estudo foram marcados cinco pontos de coleta ao longo do percurso do arroio. O primeiro ponto (P1) localizava-se próximo à nascente onde se inicia $\mathrm{o}$ fluxo corrente de água, enquanto, o último ponto de coleta (P5) estava por ficou locado onde ocorre a deságua do arroio no rio Fão. Os demais pontos foram escolhidos de acordo com a acessibilidade do local, equidistantes em média 1.000 m entre si. Em cada um dos pontos de coleta foram percorridos 100 $\mathrm{m}$ de extensão seguindo o curso do rio, adentrando na mata até $3 \mathrm{~m}$ em ambas as margens. Com relação às condições de conservação da vegetação, observou-se que no primeiro ponto de coleta (P1, 


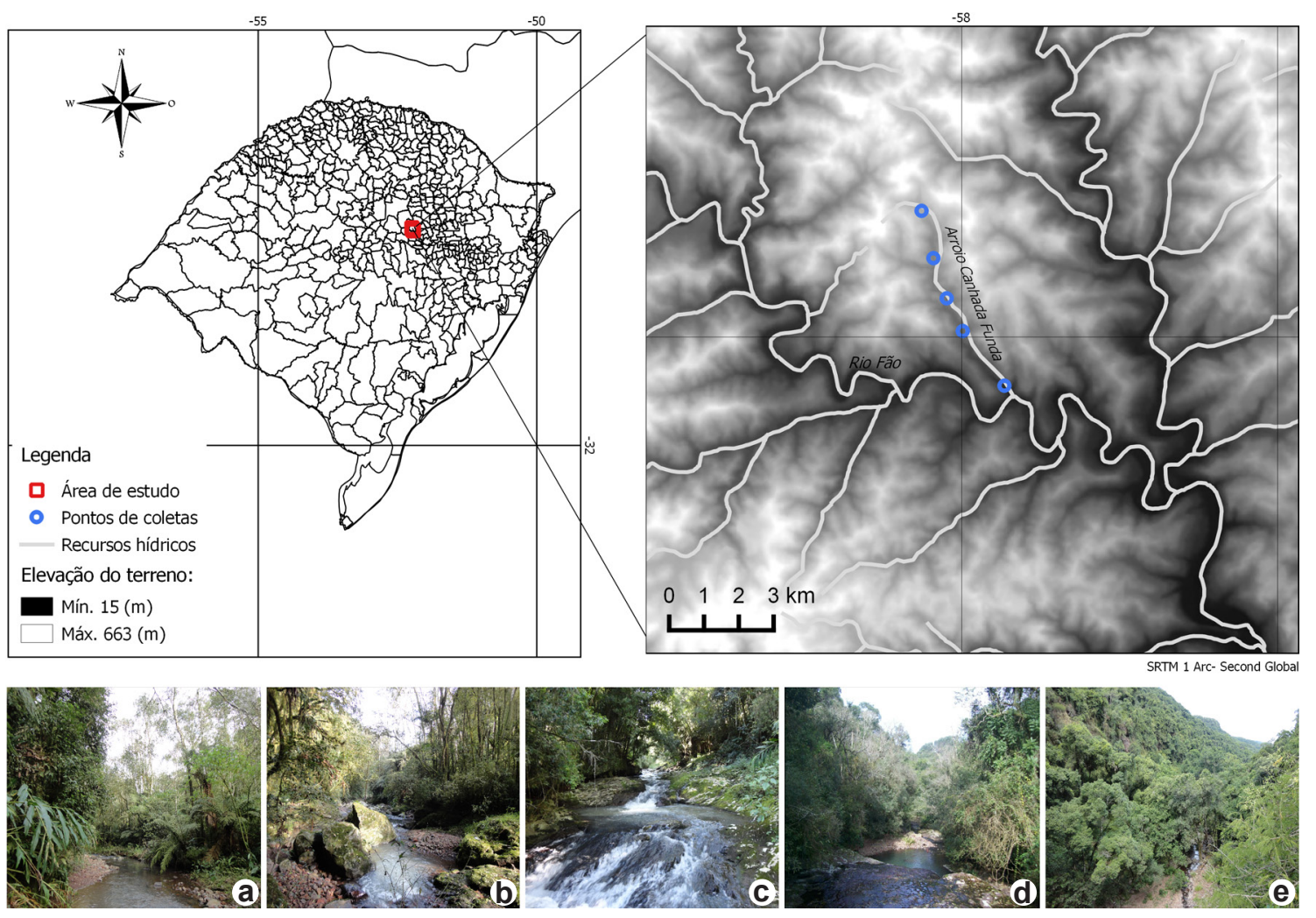

Figura 1 - a-e. Localização do Arroio Canhada Funda, Pouso Novo, Rio Grande do Sul, Brasil - detalhes dos ambientes de coleta - a,b. mata ciliar reduzida e degradada por alterações antrópicas referem-se aos pontos P1 e P2 respectivamente; c-e. vegetação preservada nos pontos de coleta mais íngremes da área P3, P4 e P5 respectivamente. Figure 1 - a-e. Location of the river Canhada Funda, Pouso Novo, Rio Grande do Sul, Brazil - details of sampling environments - a,b. riparian forest reduced and degraded by anthropic alterations refer the point P1 and P2 respectively; c-e. vegetation preserved at the steeper collection points of the area P3, P4 e P5 respectively.

altitude de $574 \mathrm{~m}$ ) a mata ciliar está muito degrada e reduzida (menos de $5 \mathrm{~m}$ de largura) devido ao uso da área para atividades agrossilvipastoris. No segundo ponto (P2, altitude de $354 \mathrm{~m}$ ), a vegetação também apresenta um elevado grau de pertubação em razão de práticas agrícolas em tempos passados, encontrando-se hoje em estágio secundário de regeneração, com dossel aberto, poucas árvores isoladas de grande porte e muitas trilhas abertas usadas por animais domésticos. No terceiro ponto de coleta (P3, altitude de $303 \mathrm{~m}$ ), a mata ciliar encontra-se em melhores condições de conservação, com a margem direita mais reduzida (10 $\mathrm{m}$ de largura), seguindo o percurso do rio, e com algumas trilhas no seu interior. Já nos dois últimos pontos (P4, altitude de $235 \mathrm{~m}$ e P5, altitude de 112 $\mathrm{m})$, devido às condições mais íngrimes do terreno, a vegetação ciliar se manteve mais conservada em mais de $30 \mathrm{~m}$ de largura em ambas as margens.

\section{Levantamento florístico}

Para as coletas foram realizadas visitas mensais à área de estudo no período de janeiro de 2012 a fevereiro de 2015 , totalizando 36 expedições. Seguindo-se o Método do Caminhamento proposto por Filgueiras et al. (1994), todo o material fértil foi coletado, identificado e classificado quanto ao hábito em Epífita (Ep), Terrícola (T) e Rupícola (R). O material testemunho foi incorporado ao Herbário PEL, Departamento de Botânica, Universidade Federal de Pelotas. A nomenclatura adotada para gêneros e categorias supragenéricas seguiu a proposta de Chase et al. (2015).

\section{Análises da composição florística}

A similaridade florística entre os cinco pontos de coleta foi observada por medidas de dissimilaridade utilizando o complemento do coeficiente de Jaccard e, posteriormente, 
efetuada uma análise de agrupamento pelo método hierárquico Neighbor-Joining. Para verificação das relações entre a variação da altitude e número de espécies observado nos pontos de coleta, foi utilizada análise de correlação linear de Pearson. A ordenação da distribuição das espécies na área de estudo foi obtida através de uma análise de especificidade composicional, que calcula a similaridade média de todas as amostras na qual ocorre cada espécie e compara o valor para a distribuição de semelhanças médias para conjuntos gerados aleatoriamente com o mesmo tamanho (Rousseeuw 1987). A especificidade composicional média de uma espécie que só ocorre uma vez é definido como 0 . Os dados foram processados e analisados através do programa estatístico $R$ ( R Core Team 2016).

\section{Resultados e Discussão}

\section{Levantamento florístico}

Na mata ciliar às margens do Arroio Canhada Funda, município de Pouso Novo, Rio Grande do Sul foram registradas 36 espécies, pertencentes a 23 gêneros de Orchidaceae (Tab. 1). Os gêneros com maior riqueza foram Gomesa R.Br. com cinco espécies, Acianthera Scheidw. e Cyclopogon C.Presl, ambos com quatro espécies, seguidos pelos gêneros Anathallis Barb.Rodr., Barbosella Schltr. e Campylocentrum Benth. representados por duas espécies cada. Os demais gêneros $(73,9 \%)$ apresentaram apenas uma espécie cada. Quanto ao hábito de vida, o epífito foi predominante, representando $69 \%$ das espécies listadas (25 spp.). O restante, 31\% das espécies (11 spp.) são terrícolas. Nenhuma espécie ocorreu exclusivamente como rupícola.

Padrões semelhantes na distribuição dos gêneros também foram observados em inventários realizados em diferentes regiões do Rio Grande do Sul (Buzatto et al. 2007; Perleberg 2009; Herbele et al. 2012). Perleberg (2009) ao avaliar a composição florística de Orchidaceae em uma localidade na Serra da Sudeste em Pelotas (RS), observou Gomesa, com sete espécies, como o mais representativo gênero, seguido de Acianthera e Anathallis, ambos com quatro espécies.

Herbele et al. (2012), ao estudarem Orchidaceae no Jardim Botânico de Lajeado (RS) em um fragmento de 20 ha, coberto por mata nativa predominantemente secundária em diferentes estádios de regeneração, listaram a presença de 27 espécies, onde Acianthera e Gomesa são apresentados como gêneros mais diversificados, com cinco e três espécies respectivamente. Embora a área do presente estudo esteja geograficamente próxima (mesma região fisiográfica) e compartilhe a mesma vegetação (Floresta Estacional Decidual) com a área estudada por Heberle et al. (2012), apenas 14 espécies foram comuns entre os dois locais, mostrando variação na composição florística, ressaltando assim, a importância de estudos pontuais para conhecer e entender os processos de distribuição das espécies em uma mesma região.

No presente estudo Cyclopogon foi um dos gêneros mais representativos com quatro espécies, enquanto nos trabalhos de Buzatto et al. (2007) e Perleberg (2009), os autores citam a presença de apenas uma e duas espécies do gênero, respectivamente. Rocha \& Waechter (2006), ao inventariarem as espécies de Orchidaceae terrícolas ocorrentes no litoral norte do estado, também relatam a representatividade de Cyclopogon (quatro spp.). Ao comparar as espécies, apenas $C$. chloroleuchus (Barb.Rodr.) Schltr. (tratado como C. polyaden (Vell.) F.S.Rocha \& Waechter por aqueles autores) é compartilhada entre as áreas estudadas, no entanto, esta espécie apresentase amplamente distribuída entre as formações vegetacionais do estado, sendo comumente relatada nos levantamentos florístico em diferentes gradientes altitudinais, inclusive em ambientes perturbados (Freitas \& Jasper 2001; Jasper et al. 2005; Perleberg 2009; Colla 2014).

O hábito de vida epífito foi predominante, assim como nos demais trabalhos realizados no estado. No entanto, a porcentagem de espécies terrícolas encontradas foi maior que as relatadas nos trabalhos de Buzatto et al. (2007), Perleberg (2009) e Herbele et al. (2012). Rocha \& Waechter (2006) relatam que aproximadamente 30\% das espécies de Orchidaceae presentes no Rio Grande do Sul são terrícolas. Os autores atribuem a elevada representatividade deste hábito, entre outros fatores, à posição subtropical e à grande área de campos do estado. Embora na área de estudo não ocorra vegetação campestre, o longo período de acompanhamento dos pontos de coleta possibilitou a amostragem de espécies que apresentam folhas expostas em determinadas épocas do ano, como é o caso de algumas espécies de Cyclopogon. Rocha \& Waechter (2006) destacam que estudos realizados em curto espaço de tempo, podem subestimar a riqueza de espécies terrícolas, pois muitas apresentam indivíduos pequenos, isolados, algumas perdendo as folhas em determinados períodos e 
Tabela 1 - Orchidaceae ocorrentes às margens do Arroio Canhada Funda, em Pouso Novo, RS, Brasil. E = epífita; $\mathrm{T}=$ terrícola; $\mathrm{R}=$ rupícola.

Table 1 - Orchidaceae occurring in river Canhada Funda in Pouso Novo, RS, Brazil. E = epiphytic; T = terrestrial; R = rupicolous.

\begin{tabular}{|c|c|c|c|}
\hline Subfamília/Tribo/Subtribo & Espécie & Hábito de vida & No PEL \\
\hline \multicolumn{4}{|l|}{ Orchidoideae } \\
\hline \multicolumn{4}{|l|}{ Cranichideae } \\
\hline \multirow[t]{7}{*}{ Spiranthinae } & Cyclopogon chloroleucus Barb.Rodr. & $\mathrm{T}$ & 26.579 \\
\hline & Cyclopogon congestus (Vell.) Hoehne & $\mathrm{T}$ & 26.580 \\
\hline & Cyclopogon elegans Hoehne & $\mathrm{T}$ & 26.581 \\
\hline & Cyclopogon warmingii (Rchb.f.) Schltr. & $\mathrm{T}$ & 26.582 \\
\hline & Eurystyles cotyledon Wawra & $\mathrm{E}$ & 26.583 \\
\hline & Mesadenella cuspidata (Lindl.) Garay & $\mathrm{T} / \mathrm{R}$ & 26.584 \\
\hline & Sarcoglottis ventricosa (Vell.) Hoehne & $\mathrm{T} / \mathrm{R}$ & 26.585 \\
\hline \multicolumn{4}{|l|}{ Orchideae } \\
\hline Orchidinae & Habenaria araneiflora Barb.Rodr. & $\mathrm{T}$ & 26.586 \\
\hline \multicolumn{4}{|l|}{ Epidendroideae } \\
\hline Tropidieae & Corymborkis flava (Sw.) Kuntze & $\mathrm{T}$ & 26.587 \\
\hline \multicolumn{4}{|l|}{ Malaxideae } \\
\hline Malaxidinae & Malaxis parthonii C.Morren & $\mathrm{T} / \mathrm{R}$ & 26.588 \\
\hline \multicolumn{4}{|l|}{ Cymbidieae } \\
\hline Catasetinae & Galeandra beyrichii Rchb.f. & $\mathrm{T}$ & 26.589 \\
\hline \multirow[t]{2}{*}{ Maxillariinae } & Maxillaria porphyrostele Rchb.f. & $\mathrm{E}$ & 26.590 \\
\hline & Maxillaria ferdinandiana Barb.Rodr. & $\mathrm{E}$ & 26.591 \\
\hline \multirow[t]{9}{*}{ Oncidiinae } & Capanemia superflua (Rchb.f.) Garay & $\mathrm{E}$ & 26.592 \\
\hline & Gomesa cornigera (Lindl.) M.W.Chase \& N.H.Williams & E & 26.593 \\
\hline & Gomesa flexuosa (Lodd.) M.W.Chase \& N.H.Williams & $\mathrm{E} / \mathrm{R}$ & 26.594 \\
\hline & Gomesa longicornu (Mutel) M.W.Chase \& N.H.Williams & $\mathrm{E}$ & 26.595 \\
\hline & Gomesa recurva $\mathrm{R} . \mathrm{Br}$ & $\mathrm{E}$ & 26.596 \\
\hline & Gomesa riograndensis (Cogn.) M.W.Chase \& N.H.Williams & $\mathrm{E} / \mathrm{R}$ & 26.597 \\
\hline & Grandiphyllum pulvinatum (Lindl.) Docha Neto & $\mathrm{E}$ & 26.598 \\
\hline & Trichocentrum pumilum (Lindl.) M.W.Chase \& N.H.Williams & $\mathrm{E}$ & 26.599 \\
\hline & Zygostates alleniana Kraenzl. & $\mathrm{E}$ & 26.600 \\
\hline \multicolumn{4}{|l|}{ Epidendreae } \\
\hline Laeliinae & Epidendrum densiflorum Hook. & $\mathrm{E} / \mathrm{R}$ & 26.601 \\
\hline \multirow[t]{9}{*}{ Pleurothallidinae } & Acianthera pubescens (Lindl.) Pridgeon \& M.W.Chase & $\mathrm{E}$ & 26.603 \\
\hline & Acianthera luteola (Lindl.) Pridgeon \& M.W.Chase & $\mathrm{E}$ & 26.604 \\
\hline & Acianthera saundersiana (Rchb.f.) Pridgeon \& M.W.Chase & $\mathrm{E}$ & 26.605 \\
\hline & Acianthera hygrophila (Barb.Rodr.) Pridgeon \& M.W.Chase & $\mathrm{E}$ & 26.606 \\
\hline & Anathallis linearifolia (Cogn.) Pridgeon \& M.W.Chase & $\mathrm{E}$ & 26.607 \\
\hline & Anathallis obovata (Lindl.) Pridgeon \& M.W.Chase & $\mathrm{E}$ & 26.608 \\
\hline & Barbosella australis (Cogn.) Schltr. & $\mathrm{E}$ & 26.609 \\
\hline & Barbosella cogniauxiana (Speg. \& Kraenzl.) Schltr. & $\mathrm{E}$ & 26.610 \\
\hline & Stelis papaquerensis Rchb.f. & $\mathrm{E}$ & 26.611 \\
\hline
\end{tabular}




\begin{tabular}{clcr}
\hline Subfamília/Tribo/Subtribo & Espécie & Hábito de vida & No PEL \\
\hline Ponerinae & Isochilus linearis (Jacq.) R.Br. & E & 26.612 \\
Calypsoinae & Govenia utriculata (Sw.) Lindl. & T & 26.613 \\
Vandeae & & & \\
Angraecinae & Campylocentrum aromaticum Barb.Rodr. & E & 26.614 \\
& Campylocentrum ulaei Cogn. & E & 26.615 \\
\hline
\end{tabular}

outras espécies são afilas durante a antese, o que pode dificultar sua localização em campo.

\section{Distribuição das espécies nos pontos de coleta}

As espécies de Orchidaceae não apresentaram distribuição uniforme ao longo da extensão do arroio. A maior riqueza (27 spp. e $26 \mathrm{spp}$.) foi observada nos pontos P4 e P5 respectivamente, enquanto que no ponto $\mathrm{P} 3$ foram registradas 18 espécies, sendo cinco exclusivas a este. Os pontos P1 e P2 apresentaram o menor número de espécies (6 spp. e 9 spp., respectivamente). Usando a análise de correlação linear de Pearson para avaliar a distribuição das orquídeas na área de estudo, observou-se uma alta correlação negativa $(r=$ $-0,8893, P<0,05)$ entre o gradiente altitudinal e a redução do número de espécies (Fig. 2).

A avaliação do componente epifítico e a flora de Orchidaceae em diferentes níveis de elevação tem apontado as variações climáticas (precipitação, temperatura e umidade) observadas ao logo do gradiente altitudinal como principais

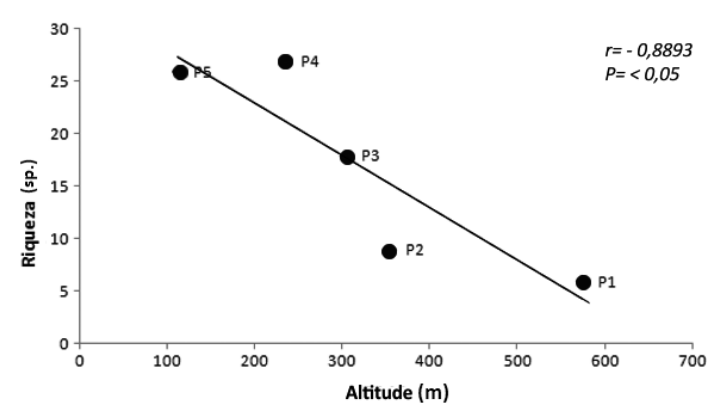

Figura 2 - Correlação de Pearson negativa entre o aumento da altitude dos pontos de coleta e redução da riqueza de espécies de Orchidaceae em mata ciliar no Arroio Canhada Funda em Pouso Novo (RS).

Figure 2 - Negative Pearson correlation between altitude increase of sampling points and reduction of Orchidaceae species richness in riparian florest in river Canhada Funda, Pouso Novo (RS). variáveis que influenciam nos padrões de riqueza e de distribuição das espécies (Hietz \& Hietz-Seifert 1995; Jacquemyn et al. 2005; Krömer et al. 2005; Acharya et al. 2011; Ding et al. 2016). Além disto, Blum et al. (2011) ao avaliarem a composição florística e distribuição de epífitas em um gradiente altitudinal (400-1.100 m) em Floresta Ombrófila Densa no Paraná, destacam também a influência do porte da vegetação e os diferentes níveis de insolação que atinge o dossel.

Embora no presente trabalho a correlação entre riqueza e altitude se mostre significativa, a variação no gradiente altitudinal analisada (112-574 m) é baixa quando comparada com os demais trabalhos discutidos anteriormente. Desta forma, a correlação observada pode estar camuflada pelo efeito da degradação, pois os pontos de maior altitude (574 e 354 m) são também, os locais onde a vegetação encontra-se mais degradada. Assim, acredita-se que ambas variáveis estejam influenciando nos resultados obtidos, sendo difícil desassociar o efeito de uma ou de outra. Conforme salientado por Rahbek (1995), os padrões de distribuição dos organismos no ambiente são afetados pela associação de diferentes variáveis (climáticas, geológicas e perturbações), as quais podem ser observadas mesmo em pequenas áreas.

Dentre as 36 espécies identificadas na área de estudo, 27 (75\%) ocorreram exclusivamente nos pontos com mata ciliar mais preservada ( $\mathrm{P} 5, \mathrm{P} 4 \mathrm{e}$ P3). Através da análise de agrupamento (Fig. 3) observa-se que estes pontos formaram um grupo, onde $\mathrm{P} 4$ e P5 mostram baixa dissimilaridade entre si (0,16 - índice de Jaccard) e P3 mostrou-se mais próximo ao $\mathrm{P} 4$, sendo compartilhadas 13 espécies entre eles. A dissimilaridade entre P1 e P2 também foi baixa $(0,2)$, formando outro agrupamento distante daquele formado pelos demais pontos. Nestes pontos, as altitudes mais elevadas e, principalmente, a redução da mata ciliar causada pelas alterações antrópicas podem desfavorecer a flora de Orchidaceae, resultando em um baixo número de espécies observado (nove spp.). 


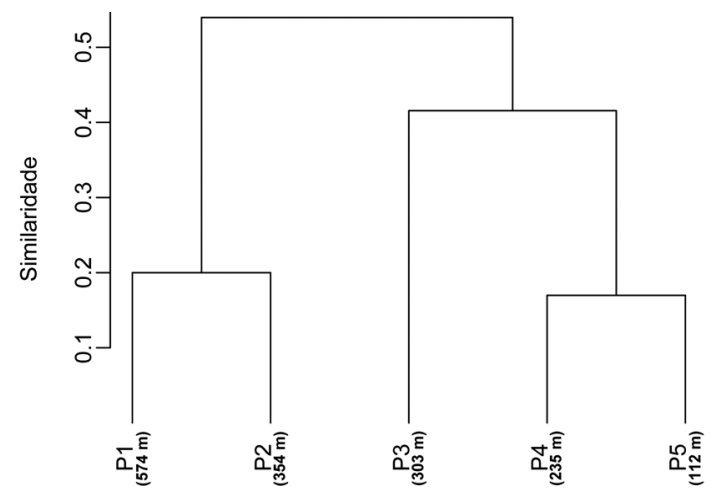

Figura 3 - Dendrograma de similaridade (índice de Jaccard) das Orchidaceae ocorrentes nos pontos de coletas de mata ciliar no Arroio Canhada Funda em Pouso Novo (RS). Entre parênteses a altitude de cada ponto de coleta. Figure 3 - Dendrogram of similarity (Jaccard index) of Orchidaceae occurring at the sampling points of riparian forest in river Canhada Funda in Pouso Novo (RS). In parentheses the altitude of each sampling point.

Adegradação florestal tende à ser inversamente proporcional a diversidade de espécies presentes em um ambiente (Barthlott et al. 2001; Bunn et al. 2010; Bataghin et al. 2017; Gomez-Días et al. 2017). A flora epifítica em florestas primárias tem se revelado distintamente mais diversa quando comparada a sítios perturbados, uma vez que as alterações antrópicas na vegetação florestal afetam as condições microclimáticas (Krömer \& Gradstein 2003; Dettke et al. 2008; Werner \& Gradstein 2009; Bataghin et al. 2010; Bataghin et al. 2017), a disponibilidade de recursos (Wolf 2005; Bonnet \& Queiroz 2006; Hietz et al. 2006) e a complexidade estrutural das florestas (Engwald et al. 2000; Barthlott et al. 2001).

Ao avaliar a composição de táxons presentes na área de estudo (Fig. 4), observa-se uma substituição de espécies ao longo dos gradientes analisados, sendo algumas espécies encontradas em todos os pontos amostrados, enquanto outras apresentam distribuição restrita a um único local de coleta. As espécies Anathalis obovata (Lindl.) Pridgeon \& M.W.Chase, Isochilus linearis (Jacq.) R.Br., Maxillaria ferdinandiana Barb. Rodr. e Stelis papaquerensis Rchb.f. ocorreram somente no último ponto (P5) onde a altitude foi menor, embora as condições de conservação da mata sejam equivalentes ao ponto anterior (P4). Acianthera pubescens (Lindl.) Pridgeon $\&$ M.W.Chase foi a orquídea mais comumente

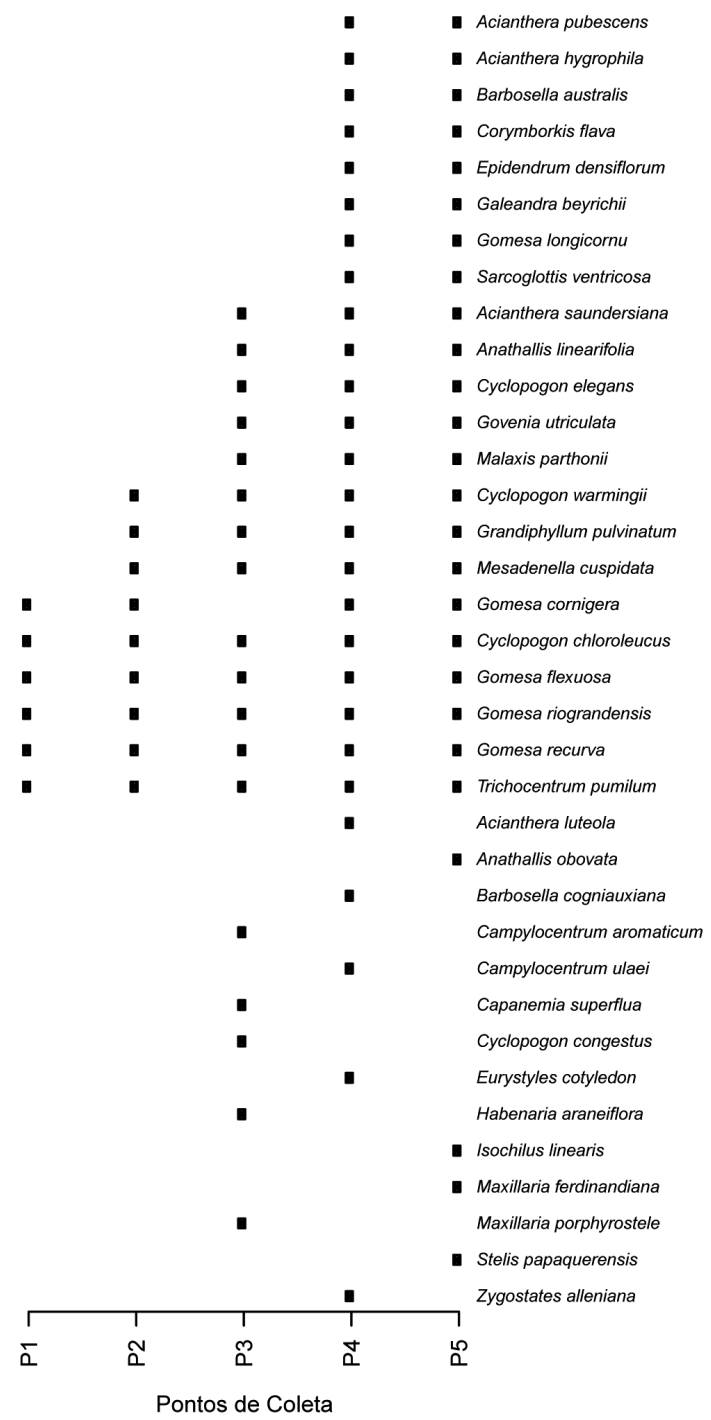

Figura 4 - Ordenação da composição das espécies de Orchidaceae registradas na mata ciliar do Arroio Canhada Funda, Pouso Novo (RS). Os pontos pretos marcam os locais de ocorrência de cada uma das espécies.

Figure 4 - Ordenation of the composition of the Orchidaceae species recorded in the riparian forest of river Canhada Funda, Pouso Novo (RS). The black point mark the occurrence sites of each species.

observada nos pontos P4 e P5. Esta espécie apresenta uma ampla distribuição geográfica, com registro de ocorrência em diferentes tipos de vegetação e altitudes (Gonçalves \& Waechter 2011; Blum et al. 2011). No entanto, para a área estudada, sua distribuição se restringiu somente aos pontos citados (P4 e P5), o que pode estar relacionado às melhores condições de conservação 
da vegetação. Barthlott et al. (2001) e Wolf (2005) em seus estudos também observaram que algumas espécies epifíticas apresentam distribuição limitada aos pontos de floresta primária, em contrapartida, algumas espécies podem apresentar adaptações que permitem o desenvolvimento também em vegetações sob diferentes graus de perturbação.

A maior parte das espécies (23 spp.) foram raramente observadas nos pontos de coleta. Para Corymborkis flava (Sw.) Kuntze e Galeandra beyrichii Rchb.f. foram registradas apenas duas populações, ambas se desenvolvendo em locais com pouca luminosidade e úmidos. C. flava apresenta ampla distribuição no estado, no entanto, sua ocorrência nunca é muito comum (Perleberg et al. 2008). Bochorny et al. (2015) destacam $G$. beyrichii como a única espécie do gênero registrada no Rio Grande do Sul, ocorrendo em áreas sombreadas do sub-bosque. Uma população de Habenaria araneiflora Barb.Rodr. foi localizada junto a uma trilha aberta próxima à borda da mata no ponto P3, estando exposta ao sol. Vale destacar a ocorrência desta espécie no local, pois a mesma é característica de formações abertas e campestres, como a maioria das espécies do gênero (Batista et al. 2013). Entre as epífitas, Acianthera luteola (Lindl.) Pridgeon \& M.W.Chase, Isochilus linearis e Eurystyles cotyledon Wawra foram encontradas somente em um local, próximas a grandes quedas de água, sugerindo a necessidade de alta umidade para seu desenvolvimento. Gonçalves \& Waechter (2011) destacam que a ecologia de muitas espécies de Orchidaceae ainda é pouco conhecida, sendo pertinentes estudos com este enfoque.

Em contrapartida, as espécies Gomesa cornigera (Lindl.) M.W.Chase \& N.H.Williams, G. flexuosa (Lodd.) M.W.Chase \& N.H.Williams, G. recurva $\mathrm{R} . \mathrm{Br}$ e Trichocentrum pumilum (Lindl.) M.W.Chase \& N.H.Williams foram comuns na área estudada, ocorrendo em todos os pontos de coletas, inclusive onde a mata encontrava-se mais perturbada. Estas espécies também são comumente citadas em levantamentos realizados em diferentes regiões do sul do Brasil, inclusive em áreas sob fortes perturbações antrópicas (Klein et al. 1978; Buzatto et al. 2007; Brustulin \& Schmitt 2008; Heberle et al. 2012; Becker et al. 2015). Em seus estudos, Moraes et al. (2015) indicam ainda $G$. cornigera e T. pumilum como espécies com maior valor de importância em áreas fragmentadas de Floresta Estacional Semidecidual no estado de São Paulo. Barros (1983) também descreve T. pumilum como uma das primeiras espécies de Orchidaceae capaz de recolonizar e se desenvolver em ambientes perturbados.

Alterações no ambiente podem causar mortalidade de indivíduos juvenis e levar à exclusão de espécies mais sensíveis, o que favorece aquelas mais resistentes, generalistas e com menores exigências de qualidade ambiental (Barthlott et al. 2001; Werner et al. 2009; Hietz et al. 2006; GómezDías et al. 2017). Ao observar a distribuição de Orchidaceae no local, verificou-se que a riqueza de espécies reduziu significativamente com o aumento da altitude. No entanto, acredita-se que a associação dos gradientes altitudinais e de perturbação da mata ciliar foram os fatores que moldaram os padrões de distribuição e composição de espécies observadas. Desta forma, a degradação da vegetação pode ser o fator mais proeminente que contribuiu para a dinâmica estrutural observada.

\section{Agradecimentos}

Agradecemos ao Laboratório de Sistemática de Fanerógamas da UFPEL, a infraestrutura e demais apoios fundamentais para o desenvolvimento deste trabalho; assim como a Yuri Feitosa e a Giselda Pereira, o auxílio nas análises; aos proprietários das áreas estudadas; às colegas Camila Gotuzzo, Ethiéne Guerra e Niziéli Cazarotto; bem como às professoras Leila Macias e Tângela Perleberg, as valiosas contribuições.

\section{Referências}

Acharya KP, Vetaas OR \& Birks HJB (2011) Orchid species richness along Himalayan elevational gradients. Journal of Biogeografy 38: 1821-1833.

Barros F (1983) Flora Fanerogâmica da Reserva do Parque Estadual das Fontes do Ipiranga: Orchidaceae. Hoehnea 10: 74-124

Bataghin FA, Barros F \& Pires JRS (2010) Distribuição da comunidade de epífitas vasculares em sítios sob diferentes graus de perturbação na Floresta Nacional de Ipanema, São Paulo, Brasil. Revista Brasileira de Botânica 33: 501-512.

Bataghin FA, Pires JS, Barros F \& Müller A (2017) Epífitas vasculares da Estação Ecológica Barreiro Rico, Anhembi, SP, Brasil: diversidade, abundância e estratificação vertical. Hoehnea 44: 172-183.

Batista JAN, Borges KS, Faria MWF, Proite K, Ramalho AJ, Salazar GA \& van den Berg C (2013) Molecular phylogenetics of the species-rich genus Habenaria (Orchidaceae) in the New World based on nuclear and plastid DNA sequences. Molecular Phylogenetics and Evolution 67: 95-109.

Barthlott W, Schmit-Neuerburg V, Nieder J \& Engwald S (2001) Diversity and abundance of vascular 
epiphytes: a comparison of secondary vegetation and primary montane rain forest in the Venezuelan Andes. Plant Ecology 152: 145-156.

Becker DFP, Padoin TOH, Nascimento CA, Robalski JL, Linden R \& Schmitt JL (2015) Riqueza e composição de epífitos vasculares em áreas urbanas da bacia hidrográfica do Rio dos Sinos, RS, Brasil. Pesquisas, Botânica 68: 227-238.

Benzing DH (1990) Vascular epiphytes: general biology and related biota. Cambridge University Press, Cambridge. 376p.

BFG - The Brazil Flora Group (2015) Growing knowledge: an overview of seed plant diversity in Brazil. Rodriguésia 66: 1085-1113.

Blum CT, Roderjan CV \& Galvão F (2011) Composição florística e distribuição altitudinal de epífitas vasculares da Floresta Ombrófila Densa na Serra da Prata, Morretes, Paraná, Brasil. Biota Neotropica 11: 141-159.

Bochorny T, Monteiro SHN \& Smidt EC (2015) O gênero Galeandra (Orchidaceae: Catasetinae) no estado do Paraná, Brasil. Rodriguésia 66: 221-227

Bonnet A \& Queiroz MH (2006) Estratificação vertical de bromélias epifíticas em diferentes estádios sucessionais da Floresta Ombrófila Densa, Ilha de Santa Catarina, Santa Catarina, Brasil. Revista Brasileira de Botânica 29: 217-228.

Brustulin J \& Schmitt JL (2008) Composição florística, distribuição vertical e floração de orquídeas epifíticas em três parques municipais do estado do Rio Grande do Sul, Brasil. Pesquisas, Botânica 59: 143-158.

Bunn WA, Jenkins MA, Brown CB \& Sanders NJ (2010) Change within and among forest communities: the influence of historic disturbance, environmental gradients, and community attributes. Ecography 33: 425-434.

Buzatto CR, Freitas EM, Silva APM \& Lima LFP (2007) Levantamento florístico das Orchidaceae ocorrentes na Fazenda São Maximiano, Município de Guaíba, Rio Grande do Sul. Revista Brasileira de Biociências 5: $19-25$.

Buzatto CR, Ferreira PPA, Seger GDS, Hertzog A \& Singer RB (2010a) O gênero Cattleya Lindl. (Orchidaceae: Laellinae) no Rio Grande do Sul. Revista Brasileira de Biociências 8: 388-398.

Buzatto CR, Singer RB \& van den Berg C (2010b) O gênero Capanemia Barb. Rodr. (Orchidaceae: Oncidiinae) na Região Sul do Brasil. Revista Brasileira de Biociências 8: 309-323.

Buzatto CR, Sanguinetti A, Romero-González GA, van den Berg C \& Singer RB (2014) A taxonomic synopsis of Brazilian Chloraeinae (Orchidaceae: Orchidoideae). Phytotaxa 158: 1-22.

Chase MW, Cameron KM, Freudenstein JV, Pridgeon AM, Salazar G, van den Berg C \& Schuiteman A (2015) An updated classification of Orchidaceae. Botanical Journal 177: 151-174.
Colla FB (2014) Distribuição e conservação de orquídeas terrestres em florestas subtropicais brasileiras. Dissertação de Mestrado. Universidade Federal do Rio Grande do Sul, Porto Alegre. 49p.

Cordeiro JLP \& Hasenack H (2009) Cobertura vegetal atual do Rio Grande do Sul. In: Pillar VDP, Müller SC, Castilhos ZMS, Jacques AVÁ (eds.) Campos sulinos - conservação e uso sustentável da biodiversidade. MMA, Brasília. Pp. 285-299.

Dettke GA, Orfrini AC, Milaneze-Gutierre MA (2008) Composição florística e distribuição de epífitas vasculares em um remanescente alterado de Floresta Estacional Semidecidual no Paraná, Brasil. Rodriguésia 59: 859-872.

Ding Y, Liu G, Zang R, Zhang J, Lu X \& Jihong Huang J (2016) Distribution of vascular epiphytes along a tropical elevational gradient: disentangling abiotic and biotic determinants. Scientific Reports 6: 1-11.

Engwald S, Schimit-Neuerburg V \& Barthlott W (2000) Epiphytes in rain forest of Venezuela - diversity and dynamics of a biocenosis. In: Breckle SW, Schweizer B \& Arndt U (eds.). Results of worldwide ecological studies. Günter Heimbach, Hoheneim.Pp. 425-434.

Filgueiras TS, Brochado AL, Nogueira PE \& Guala GF (1994) Caminhamento - um método expedito para levantamentos florísticos qualitativos. Caderno de Geociências 12: 39-43.

Freitas EM \& Jasper A (2001) Avaliação da flora Orchidaceae em uma porção de Floresta Estacional Decidual no município de Lajeado, Rio Grande do Sul. Pesquisas, Botânica 51: 113-127.

Giongo C \& Waechhter JL (2004) Composição florística e estrutura comunitária de epífitos vasculares em uma floresta de galeria na Depressão Central do Rio Grande do Sul. Revista Brasileira de Botânica 27: 563-572.

Gómez-Díaz JA, Krömer T, Carvajal-Hernández CI, Gerold G \& Heitkamp F (2017) Richness and distribution of herbaceous angiosperms along gradients of elevation and forest disturbance in central Veracruz, Mexico. Botanical Scienses 95: $1-22$.

Gonçalves CN \& Waechter JL (2011) Sinopse do gênero Acianthera Scheidw. (Orchidaceae) no Rio Grande do Sul, Brasil. Revista Brasileira de Biociências 9: 143-155.

Heberle W, Freitas EM \& Jasper A (2012) A família Orchidaceae no Jardim Botânico de Lajeado, Rio Grande do Sul, Brasil. Pesquisas, Botânica 3: 189199.

Hietz P \& Hietz-Seifert U (1995) Composition and ecology of vascular epiphyte communities along an altitudinal gradient in central Veracruz, Mexico. Journal of Vegetation Science 6: 487-498.

Hietz P, Buchberger G \& Winkler M (2006) Effect of forest disturbance on abundance and distribution of epiphytic bromeliads and orchids. Ecotropica 12: 103-112. 
IBGE, Instituto Brasileiro de Geografia e Estatística. (2011) Mapas de Biomas do Brasil. Disponível em <http://www.ibge.gov.br/home/presidencia/ noticias/21052004biomashtml.shtm $>$. Acesso em 21 outubro 2014.

Jacquemyn H, Micheneu C, Robertes DL \& Paileer T (2005) Elevational gradients of species diversit, breeding system and floral traits of orchid species on Réunion Island. Journal of Biogeography 32: 1751-1761.

Jasper A, Freitas EM, Musskopf EL \& Bruxel J (2005) Metodologia de salvamento de Bromeliaceae, Cactaceae e Orchidaceae na pequena central hidrelétrica (PCH) Salto Forqueta - São José do Herval/Putinga - RS - Brasil. Pesquisas, Botânica 56: 265-284.

Johansson D (1974) Ecology of vascular epiphytes in West African rain forest. Acta Phytogeographyca Suecica 59: 1-136.

Klein RM, Bresolin A \& Reis A (1978) Distribuição de orquídeas da Ilha de Santa Catarina e arredores. Insula 9: 3-29.

Krömer T \& Gradstein R (2003) Species richness of vascular epiphytes in two primary forests and fallows in the bolivian Andes. Selbyana 24: 190-195.

Krömer T, Kessler M, Gradstein SR \& Acebey A (2005) Diversity patterns of vascular epiphytes along an elevational gradient in the Andes. Journal of Biogeography 32: 1799-1809.

Moraes CP, Souza-Leal T, Favetta MMS, Sommer JR, Prezzi LE, Canonici TF \& Junior OR (2015) Floristic and ecology of Orchidaceae in semideciduous seasonal forest fragment in Araras, São Paulo, Brazil. Revista em Agronegócio e Meio Ambiente 8: 665-681.

Pedron M, Buzatto CR, Ramalho AJ, Carvalho BM, Radins JA, Singer RB \& Batista JAN (2014) Molecular phylogenetics and taxonomic revision of Habenaria section Pentadactylae (Orchidaceae, Orchidinae). Botanical Journal of the Linnean Society 175: 47-73.
Perleberg DP, Hentschke GS \& Singer RB (2008) O gênero Corymborkis Thouars (Orchidaceae: Tropidieae) no Rio Grande do Sul, Brasil. Revista Brasileira de Biociências 6: 375-379.

Perleberg TD (2009) A família Orchidaceae no Morro Quilongongo, Pelotas, Rio Grande do Sul, Brasil. Dissertação de Mestrado. Universidade Federal de Pelotas, Pelotas. 157p.

R Development Core Team (2011) R: a language and environment for statistical computing. R Foundation for Statistical Computing, Vienna, Austria. Disponível em <http://www.R-project. org $>$. Acesso em 12 janeiro 2017.

Rahbek C (1995) The elevational gradient of species richness: a uniform pattern? Ecography 18: 200-205.

Rocha FS \& Waechter JL (2006) Sinopse das Orchidaceae terrestres ocorrentes no litoral norte do Rio Grande do Sul, Brasil. Acta Botanica Brasilica 20: 71-86.

Rogalski JM \& Zanin EM (2003) Composição florística de epífitos vasculares no estreito de Augusto César, Floresta estacional decidual do Rio Uruguai, RS, Brasil. Revista Brasileira de Botânica 26: 551-556.

Rousseeuw PJ (1987) Silhouettes: a graphical aid to the interpretation and validation of cluster analysis. Journal of Computational and Applied Mathematics 20: 53-65.

Vogel HF, Zawadzki CH \& Metri R (2009) Florestas ripárias: importância e principais ameaças. Revista de Saúde e Biologia 4: 24-30.

Waechter JL (1998) Epiphytic orchids in eastern subtropical South America. In: 15 world orchid conference, Rio de Janeiro. Proceedings. Naturalia, Turriers. Pp. 332-341.

Werner FA \& Gradstein SR (2009) Diversity of dry forest epiphytes along a gradient of human disturbance in the tropical Andes. Journal of Vegetation Science 20: $59-68$.

Wolf JHD (2005) The response of epiphytes to anthropogenic disturbance of pine-oak forests in the highlands of Chiapas, Mexico. Forest Ecology and Management 212: 376-393. 